\title{
Laser Tool for Single Cell Transfer
}

\author{
D. Riester ${ }^{1}$, A. Özmert ${ }^{1}$, M. Wehner ${ }^{1}$ \\ ${ }^{*}$ Fraunhofer Institute for Laser Technology, Steinbachstr. 15, 52074 Aachen, Germany
}

\begin{abstract}
Many structures in living organisms show a high complexity. One example is the stem cell niche, where different cell types interact in a highly defined manner. Such structures need a building technology, which enables a highly defined and reproducible positioning of the cell types of interest. Laser Induced Forward Transfer (LIFT) however enables us to achieve a very good cell transfer even on a single cell resolution. As a test system for cell transfer polystyrene particles with a diameter of $10 \mu \mathrm{m}$ and a cell-like density were used. Single particles embedded in Matrigel $^{\mathrm{TM}}$ are reproducibly deposited onto a receiver in a pre-set pattern. Cell transfer is performed thereafter with mouse fibroblast 3T3 cells. Results are comparable to particle transfer experiments. Viability of these cells is tested with Life/Dead staining after three hours and six days. Further, proliferation of the cell line is observed after six days of cell incubation.
\end{abstract}

DOI: $10.2961 /$ jlmn.2014.02.0003

Keywords: LIFT, eukaryotic cells, single cell transfer, laser tool, hydrogel

\section{Introduction}

Recent years show an increased interest in stem cell research and their application in medicine, since they represent a unique type of mammalian cells. Their function is twofold. They are capable to differentiate into different cell types thus giving rise to all cells of the body. Therefore they are important for body regeneration. Furthermore they also keep themselves in a quiescent state hence allowing a lifelong healing ability. They serve as the source for body regeneration and maintenance $[1 ; 10]$.

Being able to understand and control these differentiation dynamics would provide entire new possibilities in basic research, disease control, tissue repair and organ regeneration. However, the stem cell's natural environment, called stem cell niche, is a complex system composed of spatially defined positions of different cell types in an extracellular matrix. Stem cell renewal, apoptosis or differentiation is determined by cell-cell communication and location within the stem cell niche [8]. Understanding of the stem cell dynamics therefore represents the key to unlock the stem cells' potential for medicine. However, in vivo research of such niches provokes ethical debates about animal experiments. Additionally such experiments are cost intensive and time consuming. An artificial stem cell niche for in vitro assays would highly accelerate basic research in this field. It would also solve ethical concerns as today animal experiments are the only way to investigate these niches. In addition, the ability to create on-demand tissue environments would solve accessibility problems of stem cell niches and has the potential to critically advance cancer research and personal medicine.

To provide such artificial tissues in vitro, a technology has to be used that is capable of exactly positioning viable cells within a three dimensional environment similar or even identical to the extra cellular matrix of in vivo stem cell niches $[7 ; 6]$.

Therefore printing technologies for living cells become more and more important $[9 ; 4]$. With state of the art ink jet printing technology it is not feasible to transfer one single cell reliably [5]. The LIFT-technology has been demonstrated to transfer cells over short distances up to $2 \mathrm{~mm} \mathrm{[2}$; 11]. The LIFT layer system consists of a setup of two glass slides called transfer slide and receiver slide. The transfer slide consists of three layers, a support layer (glass) an absorption layer ( $80 \mathrm{~nm}$ titanium) and a transfer layer (see 2.2). One laser pulse is triggered while focusing on the absorption layer. A vapor bubble is generated in the transfer layer and its expansion propels the transfer layer forward [3]. Hence, the embedded particle or cell is transferred onto the receiver slide.

As a first step towards artificial stem cell niche generation, this work analyzes the effect of process parameters such as laser fluence on the transfer of two different matrix systems, Matrigel ${ }^{\mathrm{TM}}$ and fibrin. Both matrices are suitable for cell culture but differ in their composition and processing. A polystyrene particle LIFT system is established to evaluate if particles may serve as a suitable substitution for cells. If this is found positive, LIFT parameters could be tested and their effects analyzed much faster compared to cells, since particles are not limited to generation time, accessibility or do not require a sterile environment. The experiments conclude with the LIFT of mammalian cells embedded into both Matrigel ${ }^{\mathrm{TM}}$ and fibrin matrix.

Since patterning of cells not only requires accurate positioning but also defined quantities of cells, dispensing single cells and particles, respectively, is another crucial issue for stem cell niche generation. The transfer of a single particle or cell therefore is a crucial feature for creating a stem cell niche. 
This work is presenting results with a microchip laser system with integrated trans-illumination monitoring system. Barron et al. have described that single cells can be transferred using LIFT. Yet single cell transfer was randomly achieved during the experiments [12]. Our experimental findings shall prove whether the installation of a particle transfer system is advantageous or not to further improve the LIFT of mammalian cells. Orthon et al. demonstrated for a $266 \mathrm{~nm}$ laser that trans-illumination is a helpful tool for controlled LIFT experiments [13]. This work is aimed to demonstrate the capability of the LIFT technology to transfer particles and cells at the ultimate single particle and cell resolution, which underlines its usefulness for the generation of artificial, cell-by-cell constructed in vitro stem cell niches.

\section{Materials and Methods}

\subsection{LIFT Setup}

A microchip laser (Crylas FTSS355-Q3, $\lambda=355 \mathrm{~nm}$, $1 \mathrm{~ns}$ pulse width, $1 \mu \mathrm{J}-10 \mu \mathrm{J} /$ pulse) is used to be focused on a transfer slide. In this work we show a tool design, which enables us to select and position cells before the transfer process (Fig. 1). Thereafter an automated positioning onto a receiver enables us to build defined patterns.

For all experiments a diode pumped Q3 $355 \mathrm{~nm}$ nanosecond pulsed laser (CryLaS GmbH, Germany) was used. Focus diameter was $20 \mu \mathrm{m}$. Repetition rate was $1000 \mathrm{~Hz}$ at a pulse width of $1 \mathrm{~ns}$. Correct setting of the focal point is checked by a coaxial optical system via trans-illumination before transfer. For LIFT of individual targets within the transfer layer, such as particle LIFT and cell LIFT, a positioning of the transfer slide into a correct position is performed (adjusted manually). To achieve this, the transfer slide is positioned in a way that the focal point of the laser lies in the absorption layer located above the individual target. This ensures that transfer only happens at locations where cells or particles are present.

\subsection{Preparation of Transfer and Receiver Slide}

The transfer and receiver slides are cleaned with $2 \%$ Hellmanex II (Hellma GmbH \& Co. KG, Germany) at $37^{\circ} \mathrm{C}$ for 30 minutes, washed with demineralized water, dried at $60{ }^{\circ} \mathrm{C}$ in the heating cabinet and sterilized with $70 \%$ ethanol. Thereafter the transfer layer is prepared as follows. For particle LIFT, red colored polystyrene particles with diameters of $10 \mu \mathrm{m} \pm 2.5 \mu \mathrm{m}$ with $\mathrm{NH} 2$-surface (micromod Partikeltechnologie GmbH, Germany) are used. $12-30 \mu \mathrm{L}$ of the particle solution (concentration $50 \mathrm{mg} / \mathrm{mL}$ ) were centrifuged, supernatant water was disposed and the pellet was re-suspended in $200 \mu \mathrm{l}$ of hydrogel. The hydrogel-particle suspension was thereafter applied on a titanium coated glass slide and evenly spread by a wire wound rod $(150 \mu \mathrm{m}$ rod size). The particle transfer slide is thereafter used as described in 2.1 .

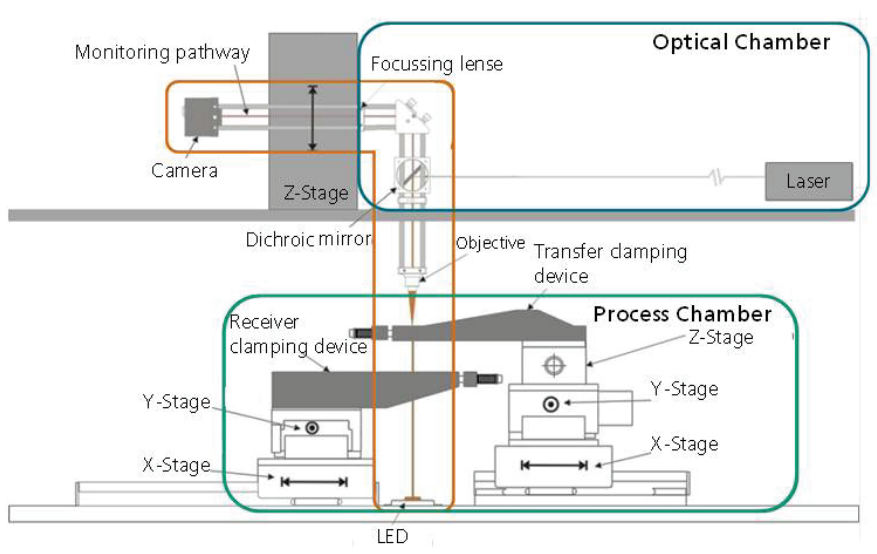

Fig. 1 Overview of LIFT Setup used for single cell transfer. A transmission illumination allows for the analysis of the transfer layer for cells.

For cell LIFT 3T3 cells are used. Cells are grown in a Modified Dulbecco's Medium with a final concentration of $10 \%$ of fetal bovine serum. For a cell LIFT target preparation $200 \mu \mathrm{l}$ of $5 \%$ Gelatin (Sigma Aldrich, Germany) are coated on a titanium coated glass slide with a wire wound $\operatorname{rod}(150 \mu \mathrm{m}$ rod size). The gel is incubated at room temperature. 8000 cells are suspended in $1 \mathrm{ml}$ medium and poured on the coated slide. After an incubation time of 30 minutes, excess medium is removed from the slide. This transfer slide is used for LIFT as described above.

The receiver slide is coated with the damper layer of a gel. The gel layer might act as a damper layer for the cells. Layer thickness was at least $500 \mu \mathrm{m}$ (estimated by used hydrogel volume).

\subsection{Receiver Slide Analysis}

After LIFT the receiver slides are analyzed by optical light microscopy. Particles can be easily detected in bright field and the hydrogel transfer can be made visible in phase contrast microscopy. Particles can be either found within one spot or in close proximity to one. Hence particles that were not within the boundaries of one spot were assigned to the closest spot.

When working with Swiss Albino 3T3 cells, a life/dead staining is performed. Therefore Calcein AM (SigmaAldrich, Germany) and Propidium Iodide (Calbiochem, Germany) are added to the sample with a final concentration of $1 \mu \mathrm{g} / \mathrm{ml}$. Cells were then incubated for 3 hours at $37^{\circ} \mathrm{C}$ with $5 \% \mathrm{CO}_{2}$. These cells are analyzed by fluoresence microscopy to check, whether the cells survived the transfer process. Further, cells are cultivated for up to 6 days to demonstrate cell divisions.

\section{Results and Discussion}

\subsection{Particle LIFT}

After laser transfer the position of the polystyrene particles were analyzed on the receiver slide with light microscopy. As soon as a LIFT transfer was performed particles were detected within the transferred spots on the receiver slides. For a laser fluence of $0.9 \mathrm{~J} / \mathrm{cm}^{2}$ the process reliabil- 
ity and position accuracy was fairly good, but at higher fluences a tendency was observed that more than one particle was transferred with one spot (see Fig. 2).
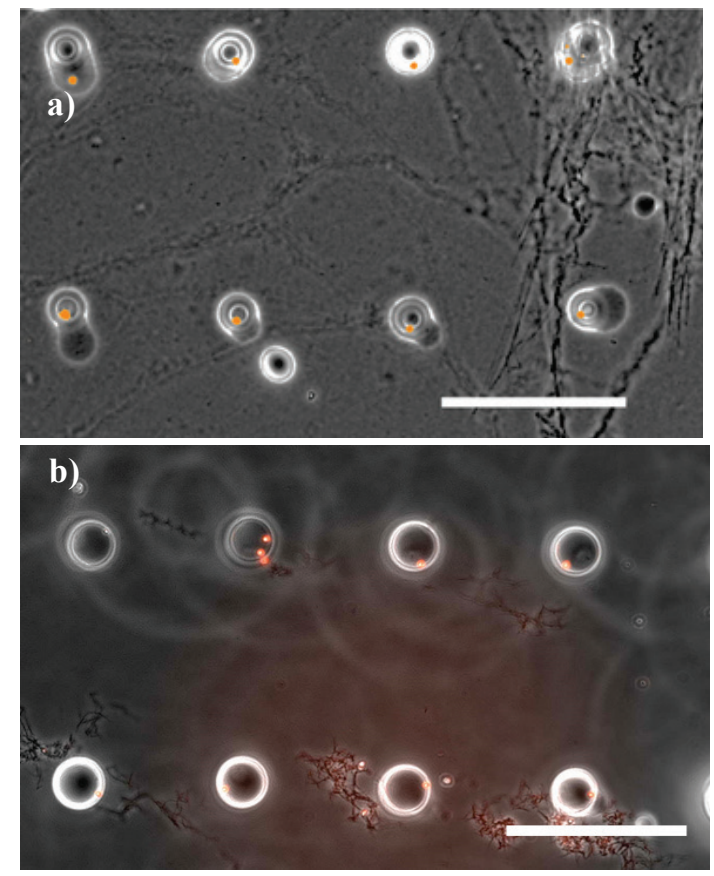

Fig. 2 Receiver slide after polystyrene particle transfer. On the receiver slide a gelatin coating with $200 \mu \mathrm{m}$ thickness is applied. Polystyrene particles are embedded in fibrin gel. When high fluences are used, the chance rises that more than one particle is transferred per spot. Scale bar: $250 \mu \mathrm{m}$ a) $0.9 \mathrm{~J} / \mathrm{cm}^{2}$; b) $1.8 \mathrm{~J} / \mathrm{cm}^{2}$

In more detail it could be observed that for fluences up to $1.2 \mathrm{~J} / \mathrm{cm}^{2}$ more than $80 \%$ of transferred spots contained one particle $(n>45)$. When higher fluences are used, the chance rises that more than one particle is transferred within one spot. This may be caused by the reason that a larger amount of hydrogel is transferred when higher fluences are used. As only one particle density was tested on the transfer slide it might be useful to reduce the particle density in the transfer layer. This may yield to a reliable single particle transfer even with higher fluences. In a case, when more hydrogel ought to be transferred per transfer this might be useful. A transfer over larger distances between transfer and receiver slide is feasible with larger volumes as drying out issues are less important. In regard to cell LIFT a low fluence transfer might be advantageous as less force is conveyed to the cell.

Additionally a comparison with inkjet printing allows for an insight about the capability of single particle LIFT. With inkjet printing the best results indicated, that in 50\% of all transfers a single cell was transferred into a well [5]. Such results are sufficient when the cell material is available in abundance. For some cells, like stem cells, a more precise deposition system is necessary. With LIFT of particles it is shown that a single particle transfer is possible in more than $80 \%$ of all spots. The result might be even improved, when the transfer material is optimized for the process regarding its viscosity and particle density. Therefore LIFT might be the technology to find out which cells are needed and which cell-cell ratio is optimal to build up in vitro stem cell niches.

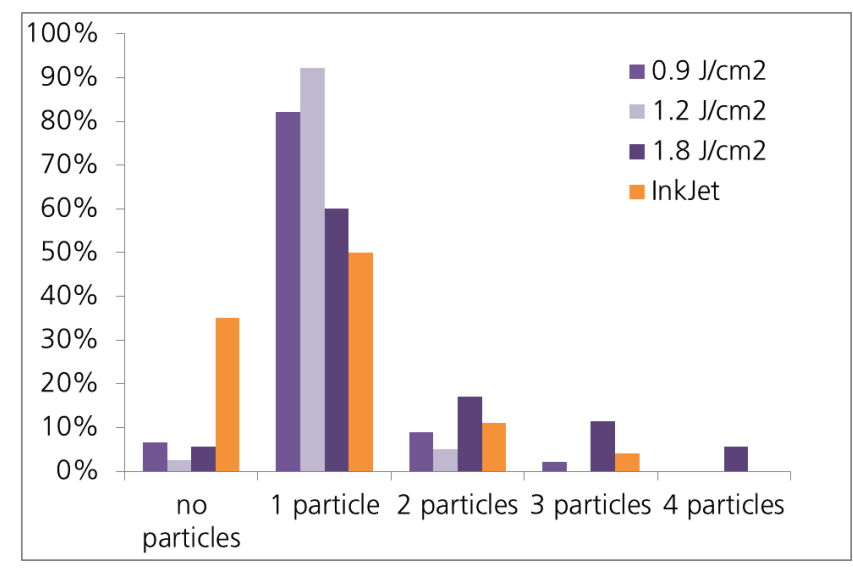

Fig. 3 Analysis of transferred particle spots for different laser fluences $(n>45)$. Inkjet results extracted from [5]

\subsection{Cell LIFT}

Results from particle LIFT showed that low transfer fluences result in a stable transfer process of single particles. Only when fluences below $1.3 \mathrm{~J} / \mathrm{cm}^{2}$ are used, surviving calls can be detected. For higher fluences, cells were transferred, but they died during the process. Weather they died due to the laser irradiation or the impact on the receiver slide has yet to be determined. Hence $1.2 \mathrm{~J} / \mathrm{cm}^{2}$ was used to transfer 3T3 mouse fibroblasts onto a Matrigel ${ }^{\mathrm{TM}}$ hydrogel. Three hours after the LIFT process a life/dead staining was performed. In several experiments it was shown that cells survive this transfer process but also dead cells can be detected. After $3 \mathrm{~h}$ a difference between surviving and nonsurviving cells is made visible by the green and red fluorescence signal. After a growing period of six days, proliferation of the $3 \mathrm{~T} 3$ cells was clearly visible (Fig. 4 a, b). This shows that cells can be transferred onto a receiver slide with a single cell resolution. To further demonstrate the capability of LIFT for single cells a pattern of four cells is printed onto a receiver slide (Fig. 4 c). Further patterns with this system are feasible and can be used for different experimental setups. 

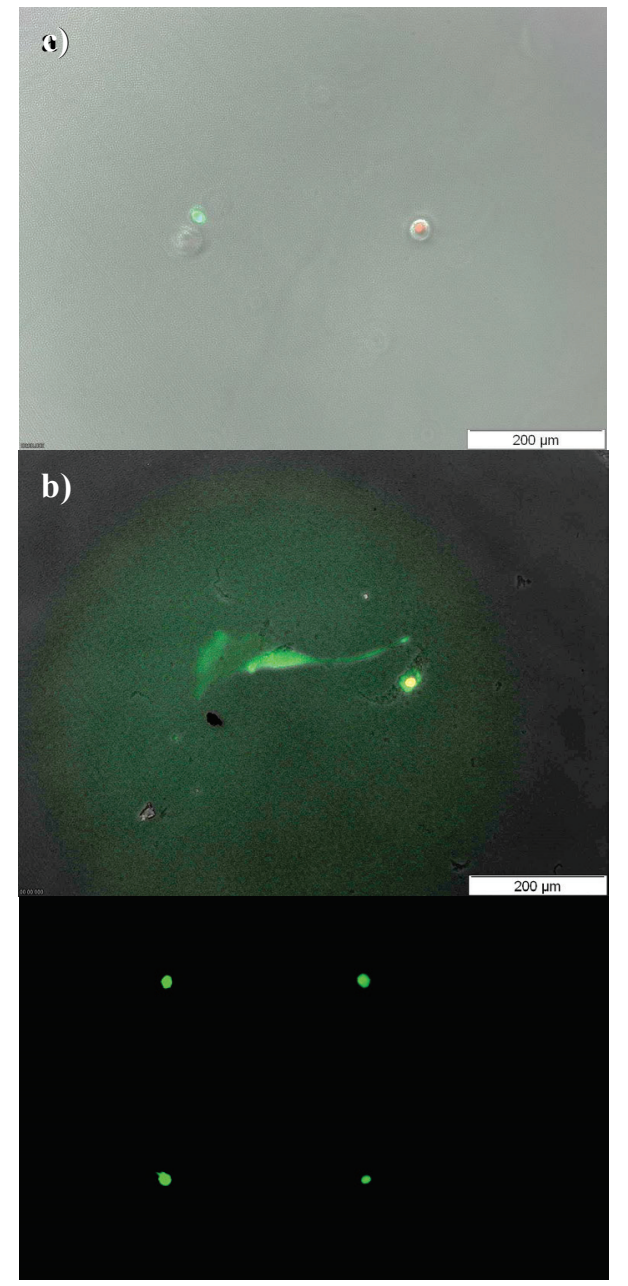

Fig. 4 Receiver slide after 3T3 cell transfer. Used fluence was $1.2 \mathrm{~J} / \mathrm{cm}^{2}$; a) $3 \mathrm{~h}$ after transfer; the green cell has survived the transfer, the red cell is dead b) $6 \mathrm{~d}$ after transfer. $3 \mathrm{~T} 3$ cells grow after single cell LIFT transfer on the receiver slide c) reproducible pattern of cells $3 \mathrm{~h}$ after transfer. Cell to cell distance is $250 \mu \mathrm{m}$

\section{Conclusion}

It is shown, that with LIFT single particles can be transferred with high precision and high reliability. This allows for a precise control of the position of these particles on the receiver slide. Thereafter the same parameters are used on Swiss Albino 3T3 cell line and the results indicate that a reproducible transfer with single cells is possible.

In this study it is systematically shown, that the LIFT system can be optimized for particle and cell transfer in a very reproducible manner. The particle LIFT system can be used to optimize parameters for following cell LIFT experiments. In future experiments the yield of surviving cells with different fluences will be determined and biological assays with different cell types will be built up.

Barron et al. and Orthon et al. have shown that cells survive at a single cell transfer level. Cell survival rates for LIFT are known to be high. Yet no data is given for the reproducibility of the single cell transfer. The number of particles and cells within one spot after LIFT has been in- vestigated in this study. It is shown that cells can be placed in a very controlled manner on a selected position.

Up until now, the laser for single cell LIFT with a transillumination monitoring setup is focused to aim for the transfer cells. It might also be an option to aim for a spot in close vicinity to the cell with higher fluences for the transfer. This may yield in a method to transfer larger connected cell cluster like embryoid bodies without inducing cell death. Further strategies for a more controlled cell transfer might be an optimization of the hydrogel damper layer on the receiver slide and a variation in the distance between transfer and receiver slide. Also the use of beam shaping tools might lead to improved single cell LIFT. A top head energy distribution might be beneficial for the jet formation process. A more stable jet leads to a higher positioning control.

By single cell LIFT it might be possible to address the requirements for creation of stem cell niches in vitro. Therefore LIFT can become an indispensable tool to generate artificial stem cell niches. This might accelerate and intensify the research and lead to new insights into stem cell function.

\section{Acknowledgments}

This work was performed in the framework of the project "ComPASS", which was funded by the Federal Ministry of Education and Research, Germany.

\section{References}

[1] Caplan, Arnold I. Journal of Cellular Physiology. (2007). 341-347.

[2] Gruene, Martin; Deiwick, Andrea; Koch, Lothar; Schlie, Sabrina; Unger, Claudia; Hofmann, Nicola; Bernemann, Inga; Glasmacher, Birgit; Chichkov, Boris. Tissue engineering. Part C, Methods (2010).

[3] Guillemot, F.; Souquet, A.; Catros, S.; Guillotin, B.; Lopez, J.; Faucon, M.; Pippenger, B.; Bareille, R.; Rémy, M.; Bellance, S.; Chabassier, P.; Fricain, J. C.; Amédée, J. Acta biomaterialia.(2010). 2494-2500.

[4] Guillotin, Bertrand; Guillemot, Fabien. Trends in Biotechnology. (2011). 183-190.

[5] Liberski, Albert R.; Delaney, Joseph T.; Schubert, Ulrich S. ACS Combinatorial Science. (2011). 190-195.

[6] Mironov, V.; Trusk, T.; Kasyanov, V.; Little, S.; Swaja, R.; Markwald, R. Biofabrication. (2009). 22001.

[7] Mironov, Vladimir; Kasyanov, Vladimir; Drake, Christopher; Markwald, Roger R. Regenerative medicine. (2008). 93-103.

[8] Moore, K. A.; Lemischka, I. R. Science. (2006). 18801885.

[9] Printed Biomaterials (Springer Science+Business Media, LLC. 2010).

[10] Orlic, Donald; Kajstura, Jan; Chimenti, Stefano; Jakoniuk, Igor; Anderson, Stacie M.; Li, Baosheng; Pickel, James; McKay, Ronald; Nadal-Ginard, Bernardo; Bodine, David M.; Leri, Annarosa; Anversa, Piero. Nature. 6829 (2001). 701-705.

[11] Schiele, Nathan R.; Corr, David T.; Huang, Yong; Raof, Nurazhani Abdul; Xie, Yubing; Chrisey, Douglas B. Bio- 
fabrication. (2010). 32001.

[12] Barron, Jason A.; Krizman, David B.; Ringeisen,

Bradley R. Annals of Biomedical Engineering. (2005).

121-130.

[13] Othon, Christina M.; Wu, Xingjia; Anders, Juanita J.;

Ringeisen, Bradley R. Biomedical materials (Bristol, Eng-

land). (2008). 34101.

(Received: August 30, 2013, Accepted: March 17, 2014) 